\title{
ANALYSIS AND SOLUTION FOR MULTI-BAND ORTHOGONAL FREQUENCY-DIVISION MULTIPLEXING ULTRA WIDE BAND SYSTEM IN REAL ENVIRONMENT FOR VEHICLE TO INFRASTRUCTURE APPLICATION
}

\author{
${ }^{1}$ Sanaa Kabil, ${ }^{3}$ Raja Elassali, ${ }^{2}$ Fouzia Elbahhar, \\ ${ }^{1}$ Abdellah Ait Ouahman and ${ }^{1}$ Brahim Ait Essaid \\ ${ }^{1}$ Department of Physics, Faculty of Science Semlalia, Cadi Ayyad University, \\ Team of Telecommunications and Computer Networks, Marrakesh, Morocco \\ ${ }^{2}$ F-59650 Villeneuve d'Ascq, Lille Nord de France University, F-59000 Lille, France \\ ${ }^{3}$ Information Technology and Modeling Laboratory, National School of Applied Sciences, \\ Cadi Ayyad University, Information Technology and Modeling, Marrakesh, Morocco
}

Received 2013-07-15, Revised 2013-08-15; Accepted 2013-08-23

\begin{abstract}
Many researches in the world deal with driving on the road to ensure optimum safety. In this context, several telecommunication technologies are often used to connect the vehicles via continuous wireless communication with the infrastructure, exchange data and information relevant to increase overall road safety and enable cooperative traffic management. These systems are not reliable for all situations and the difficulties encountered by the drivers. We have been proposed the Multi-band Orthogonal FrequencyDivision Multiplexing system based on Ultra Wide Band (MB-OFDM UWB) as a good candidate to insure a Vehicle to Infrastructure (V2I) communication with high data rate and good performances in terms of Bit Error Rate (BER). However, Inter-Carrier Interference (ICI) resulting from Doppler shift phenomenon degrades MB-OFDM UWB system performance and raises a significant challenge in wireless mobile environment. In this study, V2I communication based on the MB-OFDM UWB technology is studied and analyzed for $200 \mathrm{Mbit} / \mathrm{s}$, over multipath channel using the IEEE802.15.4a channel model with Doppler shift for different speeds. The theoretical formulas of BER in Additive White Gaussian Noise (AWGN) and Rayleigh channels are calculated and are compared to the simulation results in MB-OFDM UWB system. The comparison shows that simulation results are consistent with theoretical formulas. The degradation in the performance of the proposed system solution becomes worse with a high speed $250 \mathrm{Km} \mathrm{h}^{-1}$, so it's necessary to use new receiver solutions. The Extended Kalman Filer (EKF) and the Maximum Likelihood (ML) estimation with ZF equalizer have been proposed and have been compared to Zero-Forcing (ZF) in order to combat the ICI effect. For high value of speed $\left(250 \mathrm{Km} \mathrm{h}^{-1}\right)$, the EKF method performs better than the ML method, better than the ZF equalizer and offers much improvement in performance in term of BER.
\end{abstract}

Keywords: MB-OFDM UWB, V2I Communication, Multipath Channel, Doppler Shift, ICI Cancellation Solutions

\section{INTRODUCTION}

The poor estimation of the safety distance to be maintained between vehicles and poor visibility in fog or heavy rain has generated a large number of accidents. Thus, a secure and reliable communication between vehicle and infrastructure is necessary. The solutions of communication technologies are proposed to ensure the implementation of system driver assistance or receiving information and to ensure the safety of motorists on the road. It will be able to

Corresponding Author: Sanaa Kabil, Department of Physics, Faculty of Science Semlalia, Cadi Ayyad University,

Team of Telecommunications and Computer Networks, Marrakesh, Morocco 
transmit the vehicle's main features: its plot, its geometry, its level of adhesion and its peculiarities. It will provide information on driving conditions related to climate (rain, wind, snow, sleet and fog) or related to traffic.

Different applications are currently used and are being developed. Moreover, the Federal Communication Commission (FCC) has recognized the importance of having a dedicated wireless spectrum for improving traffic safety and highway efficiency (Bai et al., 2012). However, they are not reliable for all situations and the difficulties encountered by the drivers. Moreover they are not very suitable for V2I communication considered in terms of: Climate, rate, Number of users, Implementation, cost.

The V2I communication can be established using the new communication technique such as the Ultra Wide Band (UWB) radio technology. In fact, The UWB communication technology is an emerging for high data rate wireless communication applications (Win et al., 2009). The FCC has allocated $7500 \mathrm{MHz}$ of spectrum from 3.1 $\mathrm{GHz}$ to $10.6 \mathrm{GHz}$ for the UWB system. It has a priori the desired benefits without suffering the limitations.

The UWB technology can be divided into two groups: Single band and Multi-Band. We have been proposed the MB-OFDM UWB as a good candidate to ensure the V2I communication, it can offer high data rate with an interesting properties. However, a problem with OFDM is that its sensitivity to the Frequency Offset (FO) between transmitter and receiver signals, which may be caused by the relative motion between transmitter and receiver and scattering environment, induces Doppler spread (Sreekanth and GiriPrasad, 2012), or by difference between the transmitter and receiver local oscillator frequency. These frequency errors normally referred to as Carrier Frequency Offset (CFO) causes the loss of orthogonality between the subcarrier and signal transmitted on each carrier are not independent to each other, leading to the Inter-Carrier Interference (ICI), so that system performance may be considerably degraded.

In this study, we evaluate the performances of MBOFDM UWB technique for Vehicle-to-Infrastructure (V2I) communication system in terms of channel propriety essentially Doppler effect. The study is organized as follows. We provide a description of existing system technologies for V2I communication and we describe the proposed MB-OFDM UWB system and give its parameters. Analyses of BER are presented to validate the proposed system performance and to discuss the impact of Doppler shift, then, we propose ZeroForcing (ZF), Maximum Likelihood estimation (ML) and Extended Kalman Filter (EKF) as solutions to reduce the Doppler shift impact in MB-OFDM UWB system.

\subsection{Existing System Technologies for Vehicle- To-Infrastructure Communication}

Intelligent Transportation Systems (ITS) that incorporate advanced technologies have been purported to offer efficiencies in tackling traffic congestion (Wang et al., 2012), also to increase the road safety and ameliorate traffic flow. The vehicle will be able to communicate information to roadway infrastructure and other vehicles (Fig. 1) that help both the vehicle itself and its drivers to correctly asses the current traffic situation. Several wireless transmission technologies for ITS have been proposed as: Digital Broadcasting system, Cellular communication standards and Dedicate Short Range Communication (DSRC).

Digital broadcasting systems such as Digital Audio Broadcasting (DAB), Digital Multimedia Broadcasting (DMB), Digital Video Broadcasting-Handheld (DVB-H) or Digital Video Broadcasting-Terrestrial (DVB-T). $\mathrm{DAB}$ is a technology to broadcast audio. DMB (or TDMB) and DVB are a standard to carry video and enriched services to the mobile user and it can offer high data rate (The DVB-T system offers a data rate capacity up to $32 \mathrm{Mbit} / \mathrm{s}$ ) (Platzner et al., 2010) and T-DMB system offers a data rate between 1.06-2.3 Mbits/s (Petkovic and Bjelica, 2007).

The cellular systems such as Global System for Mobile Communication (GSM) is mature technology, always-on connectivity, however, it cannot support high-data transmission effectively (data rate up to $12 \mathrm{kbps}$ ) (Platzner et al., 2010), General Packet Radio Service (GPRS) and Universal Mobile Communication System (UMTS), can provide always-on packet-switched connectivity and were designed for high-speed data rates (GPRS can provide data rate, up to $144 \mathrm{kbps}$ (Sharma et al., 2010) and 2 Mbits for UMTS (Chantaksinopas et al., 2012).

In 1999, the U.S. Federal Communication Commission has allocated $75 \mathrm{MHz}$ of Dedicated ShortRange Communication (DSRC) spectrum at $5.9 \mathrm{GHz}$ to be used exclusively for Vehicle-to-Vehicle and Vehicleto-Infrastructure communications (Fawaz et al., 2010). DSRC standard for vehicular communications was approved by ASTM International and has been standardized by IEEE as 802.11 p (Alvin, 2011). The first purpose is to enable public safety applications. Private services are also permitted because it lowering cost and also to encourage DSRC development and adoption. It is meant to be a complement to cellular communications by providing very high data transfer ranging from 10 to $50 \mathrm{Mbits} / \mathrm{s}$. 


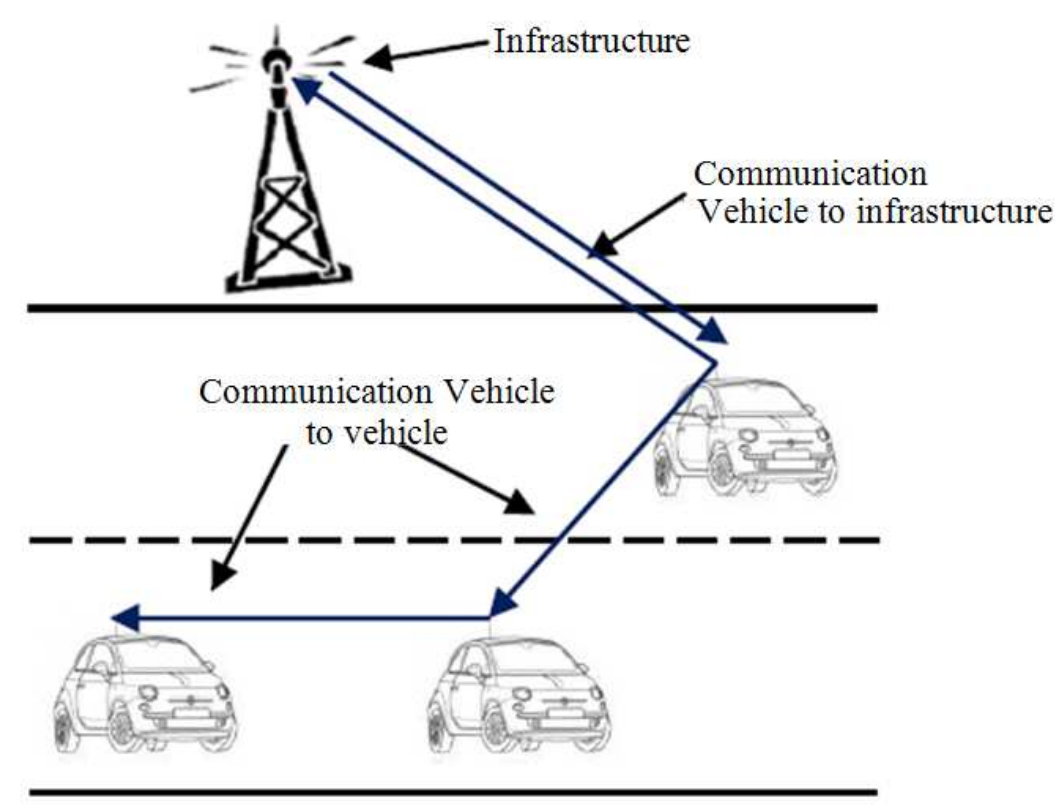

Fig. 1. Example of Vehicle-to-Vehicle, Vehicle-to Infrastructure Communications

All these technologies have advantages and disadvantages depending on the type of application and considered scenario. In our application, we need a wireless technology that can ensure sharing information's video for V2I communication with a high data rate over $100 \mathrm{Mbit} / \mathrm{s}$, so the wireless transmission technologies described above are not very suitable to satisfy this need. We have been proposed the MB-OFDM UWB system to ensure V2I communication for some reasons, the proposed system supports very high data-rate transmission (offers 480 Mbit/s data rate with QPSK modulated symbols on each subcarrier), it is attractive radio techniques that coexist with other narrowband signals and have interesting properties, provide a much more efficient in multipath channel, it reduces the complexity of the design and lowering cost.

\section{MATERIALS AND METHODS}

\subsection{MB-OFDM UWB Solution}

The UWB was originally used in radars to wireless communications and high rates over short distances for intra-building applications (Bahai et al., 2004). Two commonly used single-band impulse radio systems are time-hopping spread-spectrum impulse radio (TH-UWB) and direct-sequence spread-spectrum impulse radio (DSUWB). The Multi-band UWB system can be classified into Multi-Band Impulse Radio (MB-IR) and the MBOFDM UWB solution (Fernandes and Wentzloff, 2010) based on the transmission of continuous OFDM signals. The available spectrum $(3.1-10.6 \mathrm{GHz})$ is divided into 14 sub-bands, each one occupying $528 \mathrm{MHz}$.

The structure of the MB-OFDM UWB solution is very similar to that of a conventional wireless OFDM physical layer, the main difference is the use of TimeFrequency Codes (TFC). Each OFDM symbol is transmitted across a band of a band group, providing frequency diversity in the system.

The block diagram of the MB-OFDM UWB transmitter is show in Fig. 2.

Different channel coding rates (using 1/3 convolution coding and puncturing), Time and Frequency domain Spreading (TDS, FDS respectively) of factor 2, are employed to generate data rates of 55, 80, 160 and $200 \mathrm{Mbit} / \mathrm{s}$. The FDS consists in transmitting twice the same information in a single OFDM symbol. TDS is obtained by repeating the same OFDM symbol over different sub-bands and hence, it results in inter-sub-band frequency diversity. The coded and padded bit stream shall be interleaved prior to modulation to provide robustness against burst errors. 


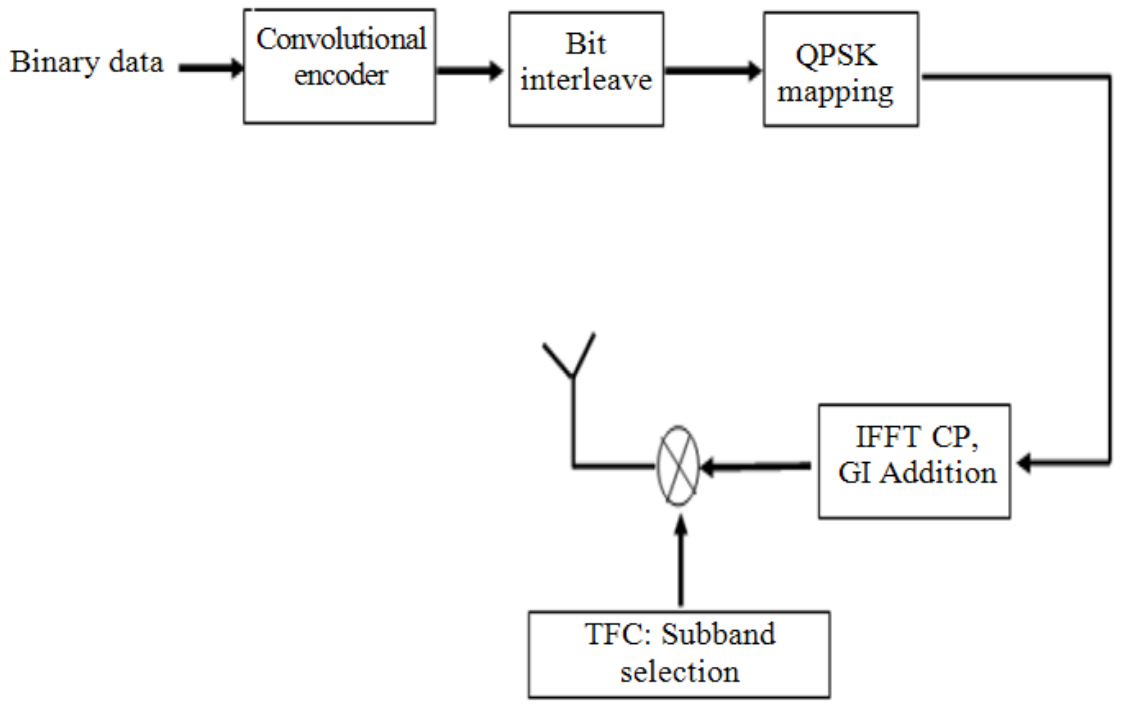

Fig. 2. Block diagrams of the transmitter for MB-OFDM UWB system

The information transmitted on each band is modulated using OFDM constructed from a Fast Fourier Transform (FFT) and modulated with digital modulation. The useful duration of each OFDM symbol is $242 \mathrm{~ns}$, leading to subcarrier frequency spacing of $\Delta \mathrm{f}=4.125$ MHz. A 128 point IFFT/FFT is used along with a short Cyclic Prefix (CP) length of $60.6 \mathrm{~ns}$ added at the end of each OFDM symbol to avoid Inter-Symbol Interference (ISI) between two consecutive OFDM symbols, it used also to mitigate multipath introduced by the channel thereby maintaining the orthogonality between the carriers received. Also, an additional guard interval of $9.5 \mathrm{~ns}$ is added to allow the transmitter and receiver to switch from one sub-band to another, a total OFDM symbol duration of $312.5 \mathrm{~ns}$ and therefore includes 165 samples.

\subsection{Theoretical Studies}

The most commonly used technique for evaluating criterions of system behaviour is the probability of BER. In order to verify the performance of MB-OFDM UWB system, we have simulated the proposed system with the following configuration described in (Fig. 2) over AWGN and Rayleigh channel models. We use the band group 1 which employs the first three sub-bands of $528 \mathrm{MHz}$, QPSK modulation, 128-point FFT/IFFT and 37 -sample Zero-Padding. Measurements have been taken for both channel models and compared with the corresponding theoretical formulas for various values of $\mathrm{E}_{\mathrm{b}} / \mathrm{N}_{0}$. Similar analytic discussions were reported in (Mohapatra and Das, 2009; Albarazi et al., 2011)

\subsection{Probability of BER over AWGN Channel}

The following theoretical formulas were concluded from (Albarazi et al., 2011). For AWGN channel model and Phase-Shift Keying (PSK) scheme, a general formula, describes the probability of bit error of M-ary PSK is:

$$
\mathrm{P}_{\mathrm{b}, \mathrm{QPSK}}=\mathrm{Q}\left(\sqrt{\frac{2 \mathrm{E}_{\mathrm{b}}}{\mathrm{N}_{0}}}\right)
$$

Where:

$\mathrm{E}_{\mathrm{b}}=$ Signal energy

$\mathrm{N}_{0}=$ Noise power spectral density

$\mathrm{Q}=$ The tail probability of Gaussian distribution, defined as:

$\mathrm{Q}(\mathrm{x})=\frac{1}{\sqrt{2 \pi}} \int_{x}^{\infty} \mathrm{e}^{-\frac{t^{2}}{2}} \mathrm{dt}$

The Equation (2) can be expressed in terms of the complementary error function as:

$$
\mathrm{Q}(\mathrm{x})=\frac{1}{2} \operatorname{erfc}\left(\sqrt{\frac{\mathrm{E}_{\mathrm{b}}}{\mathrm{N}_{0}}}\right)
$$

From the Equation 1 and 3, $\mathrm{P}_{\mathrm{b}, \mathrm{QPSK}}$ can be expressed as:

$$
\mathrm{P}_{\mathrm{b}, \mathrm{QPSK}}=\frac{1}{2} * \operatorname{erfc}\left(\sqrt{\frac{\mathrm{E}_{\mathrm{b}}}{\mathrm{N}_{0}}}\right)
$$


Figure 3 shows the probability of BER in both theoretical and simulated results for QPSK over the AWGN channel model. $\mathrm{E}_{\mathrm{b}} / \mathrm{N}_{0}$ is varying between 0 and $30 \mathrm{~dB}$.

Comparing the simulation results of BER versus $\mathrm{E}_{\mathrm{b}} / \mathrm{N}_{0}$ for uncoded MB-OFDM system over AWGN channel, with the corresponding theoretical formula described in Equation (4), we find that the performance of the proposed system perfect agrees with the analytical performance.

\subsection{Probability of BER over Rayleigh Channel}

Similar to the probability of BER over AWGN channel, the Rayleigh channel is to be validated in terms of the probability of BER. The general formula for the probability of REB of QPSK modulation over Rayleigh channel, derived in (Mohapatra and Das, 2009):

$$
\mathrm{P}_{\mathrm{b}, \mathrm{QPSK}}=\frac{1}{2}-\frac{1}{2 \sqrt{1+\frac{1}{\left(\frac{\mathrm{E}_{\mathrm{b}}}{\mathrm{N}_{0}}\right)}}}
$$

Figure 4 shows the BER performances of the uncoded MB-OFDM UWB system simulated results compared to theoretical formulas for QPSK over Rayleigh channel. $\mathrm{E}_{\mathrm{b}} / \mathrm{N}_{0}$ is varying between 0 and $30 \mathrm{~dB}$.

Comparing the simulation results of BER over Rayleigh channel, with the theoretical formula described in Equation (5), we find that the performance of the simulated proposed system agrees with analytical performance for QPSK modulation. As shown in Fig. 4 and 5 the MB-OFDM UWB system model is valid to be used to analyze the impact of radio channel environment and specially multipath with Doppler Shift effects.

\subsection{Doppler Shift Impact Analyses}

For the V2I communication, the mobility can be very high speed; therefore it is interesting and challenging to investigate the performance of the MB-OFDM UWB system in mobile radio channel.

Consider an OFDM signal represented in the time domain as in Equation (6):

$$
\mathrm{x}(\mathrm{t})=\sum_{\mathrm{k}} \mathrm{x}_{\mathrm{k}} \mathrm{e}^{\mathrm{j} 2 \pi \mathrm{f}_{\mathrm{k}} \mathrm{t}}, 0 \leq \mathrm{t} \leq \mathrm{T}
$$

$\mathrm{x}_{\mathrm{k}}$ is the complex signal modulating for the kth subcarrier, $f_{k}=f_{0}+k \Delta f$ is the frequency of the kth subcarrier, $\mathrm{f}_{0}$ is the starting frequency of the MB-OFDM signals and $\Delta \mathrm{f}$ is the frequency separation between two adjacent subcarriers.
The complex baseband representation of the time variant impulse response model of the multipath channel in mobile radio environments is defined as (Zhao and Haggman, 1996):

$$
\mathrm{h}(\mathrm{t}, \tau)=\sum_{\mathrm{i}=0}^{\infty} \alpha_{\mathrm{i}}(\mathrm{t}) \mathrm{e}^{\mathrm{j} 2 \pi \mathrm{f}_{\mathrm{d}, \mathrm{k}} \mathrm{t}} \delta\left(\mathrm{t}-\tau_{\mathrm{i}}\right)
$$

With $\alpha_{\mathrm{i}}$ is the complex multipath gain and $\tau_{\mathrm{i}}$ is the path delay:

$$
\mathrm{f}_{\mathrm{d}, \mathrm{k}}=\left(\mathrm{f}_{0}+\mathrm{k} \Delta \mathrm{f}\right) \frac{\mathrm{v}}{\mathrm{c}} \cos \theta_{\mathrm{i}}
$$

\section{Where:}

$\mathrm{f}_{\mathrm{d}, \mathrm{k}}=$ The Doppler shift of the kth subcarrier received from the ith scatter in the direction $\theta_{\mathrm{i}}$

$\mathrm{v}=$ The speed difference between the source and receiver 5

$\mathrm{C}=$ The speed of propagation

We propose a multipath channel model with two paths (Fig. 5) and with the shadowing effect. The model of Saleh-Valenzuela (SV) has been adopted as the reference model of Outdoor UWB channel specified in the IEEE802.15.4a. The model has been extracted from measurements that cover the range of $5-17 \mathrm{~m}, 3-6 \mathrm{GHz}$ as shown in (Molisch et al., 2004). We will use this model to calculate the multipath gains and the path delays.

We assume that we have the same Doppler shift for the two paths with the presence of the shadowing effect $X=2$, the Equation (7) becomes Equation (9):

$$
\mathrm{h}(\mathrm{t}, \tau)=\mathrm{e}^{\mathrm{j} 2 \pi \mathrm{f}_{\mathrm{d}, \mathrm{k}} \mathrm{t}}\left(\mathrm{X} \sum_{\mathrm{i}=1}^{2} \alpha_{\mathrm{i}}(\mathrm{t}) \delta\left(\mathrm{t}-\tau_{\mathrm{i}}\right)\right)
$$

The received signal is Equation (10):

$$
y(t)=e^{j 2 \pi f_{d, k} t}\left(X \sum_{i=1}^{2} \alpha_{i} \sum_{k} x_{k} e^{j 2 \pi f_{k}\left(t-\tau_{i}\right)}\right)+w(t)
$$

If the $y(t)$ is sampled by Nyquist rate, the Equation (10) becomes Equation (11):

$$
y(n)=e^{j 2 \pi f_{d, k} \frac{n T}{N}}\left(X \sum_{i=1}^{2} \alpha_{i} \sum_{k} x_{k} e^{j 2 \pi f_{k}\left(\frac{n T}{N}-\frac{n}{N} \tau_{i}\right)}\right)+w(n)
$$


Sanaa Kabil et al. / Journal of Computer Science 9 (10): 1305-1317, 2013

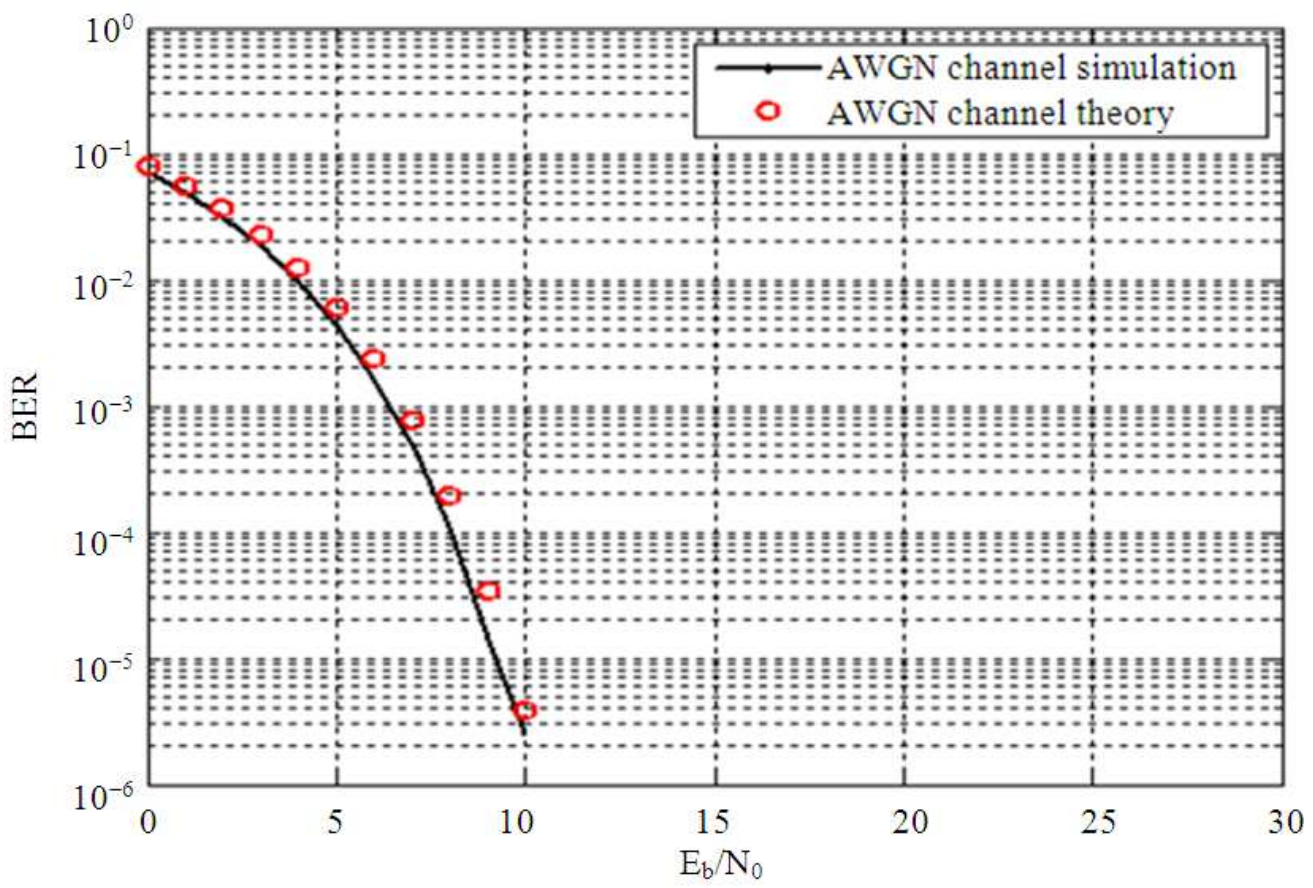

Fig. 3. Comparison of the theoretical formula of BER versus $E_{b} / N_{0}$ and the simulated MB-OFDM UWB system over AWGN channel

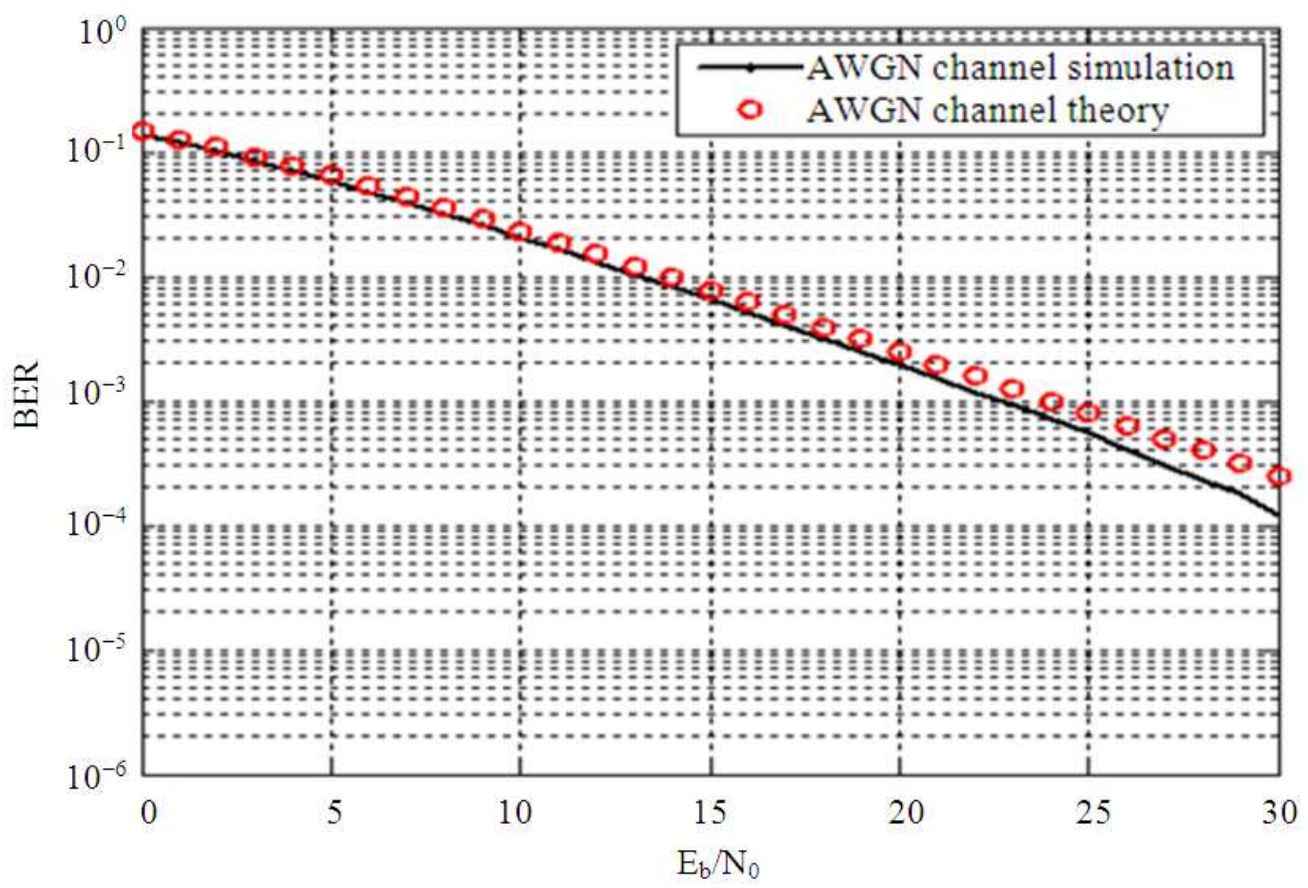

Fig. 4. Comparison of the theoretical formula of BER versus $E_{b} / N_{0}$ and the simulated MB-OFDM UWB system over Rayleigh channel 


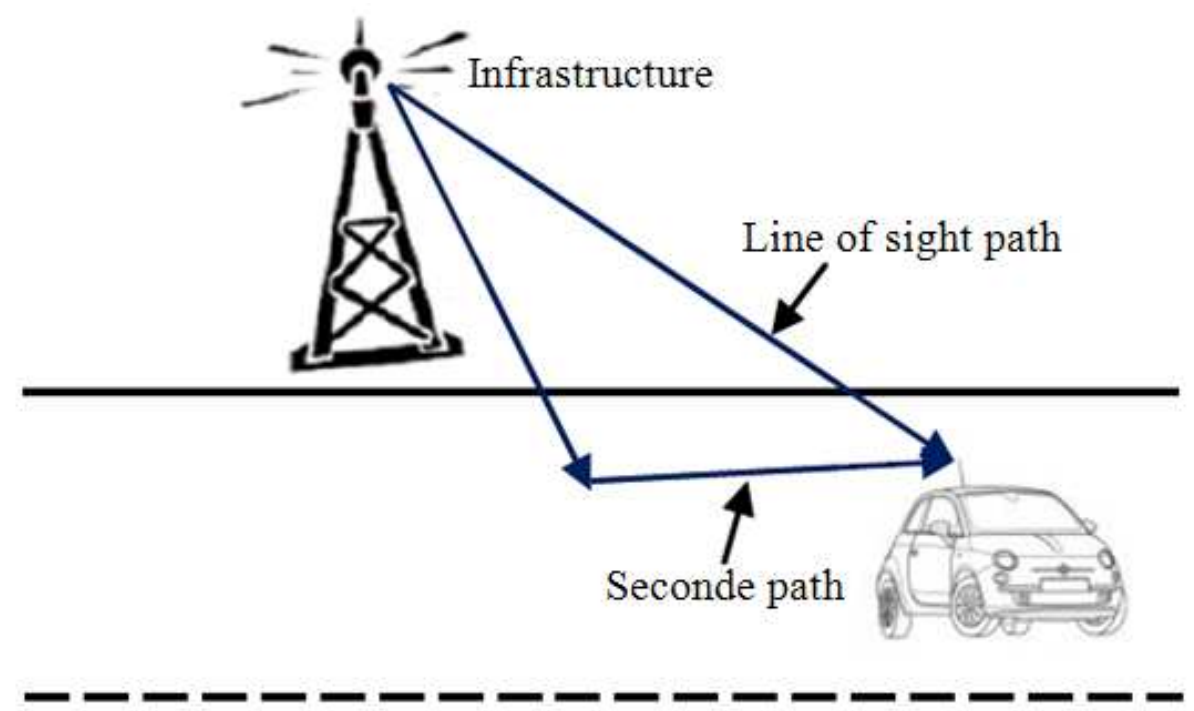

Fig. 5. Proposed multipath channel model with two-paths

The normalized Frequency Offset $(F O)$ is $\varepsilon=f_{d, k} T$. It is a more efficient parameter when analyzing frequency offset in OFDM systems.

The received signal $\mathrm{y}(\mathrm{n})$ becomes Equation (12 to 14):

$$
\begin{aligned}
& \left.y(n)=e^{j 2 \pi s \frac{n}{N}}\left(X \sum_{i=1}^{2} \alpha_{i} \sum_{k} x_{k} e^{j 2 \pi f_{k}\left(\frac{n T}{N}-\frac{n}{N} \tau_{i}\right.}\right)\right)+w(n) \\
& y(n)=z(n) e^{j 2 \pi \varepsilon \frac{n}{N}}+w(n) \\
& z(n)=x(n)\left(X \sum_{i=1}^{2} \alpha_{i} e^{-j 2 \pi f_{k} \frac{n}{N} \tau_{i}}\right)
\end{aligned}
$$

The simulation results are given in terms BER to evaluate the MB-OFDM UWB system. We simulated the band group 1, 5/8 coding rate, TDS of factor 2, are employed to generate data rates of $200 \mathrm{Mbit} / \mathrm{s}$, with QPSK modulation, 128 point FFT/IFFT and 37-samples Zero- Padding. The data rate $200 \mathrm{Mbit} / \mathrm{s}$ was plotted and tested to compare their performances and robustness over the proposed multipath channel with Doppler shift.

Figure 6 shows the simulation results of BER performance versus $E_{b} / N_{0}$ for $200 \mathrm{Mbit} / \mathrm{s}$ over the proposed multipath channel, comparing to the simulation results over the proposed multipath channel in the presence of Doppler Shift for speeds V $=[50,150,250]$ $\mathrm{Km} \mathrm{h}^{-1}$. The $\mathrm{E}_{\mathrm{b}} / \mathrm{N}_{0}$ is varying between -6 and $50 \mathrm{~dB}$.
According to the figure below, we note that the BER for the $200 \mathrm{Mbit} / \mathrm{s}$ increases as speed increase which leads an important degradation in the performance of the proposed system, this degradation becomes worse for 150 and $250 \mathrm{Km} \mathrm{h}^{-1}$. The Introduction of the ICI is destructive effect caused by CFO in MB-OFDM UWB system. So there is a need to reduce the ICI effect.

\subsection{ICI Reduction Methods for MB-OFDM UWB System}

The issue of ICI cancellation has been widely studied in digital communication systems. Recently several techniques have been proposed and developed to estimate and adjustment of the ICI effect. These techniques include the frequency domain equalization (Kumar and Pandey, 2013), the time domain windowing (Kumar and Pandey, 2013), the ICI selfcancellation schemes (Rahman et al., 2012), the use of Kalman Filter offset estimation and cancelation (Kumar et al., 2009), Maximum Likelihood Estimation (Kumar et al., 2009), in (Mohapatra and Das, 2009) they proposed a novel method called MZPSK modulation. In this section of the article, we investigate three methods for combating the ICI effect: Zero-Forcing (ZF) equalizer, Maximum Likelihood estimation (ML) and Extended Kalman Filter (EKF). 


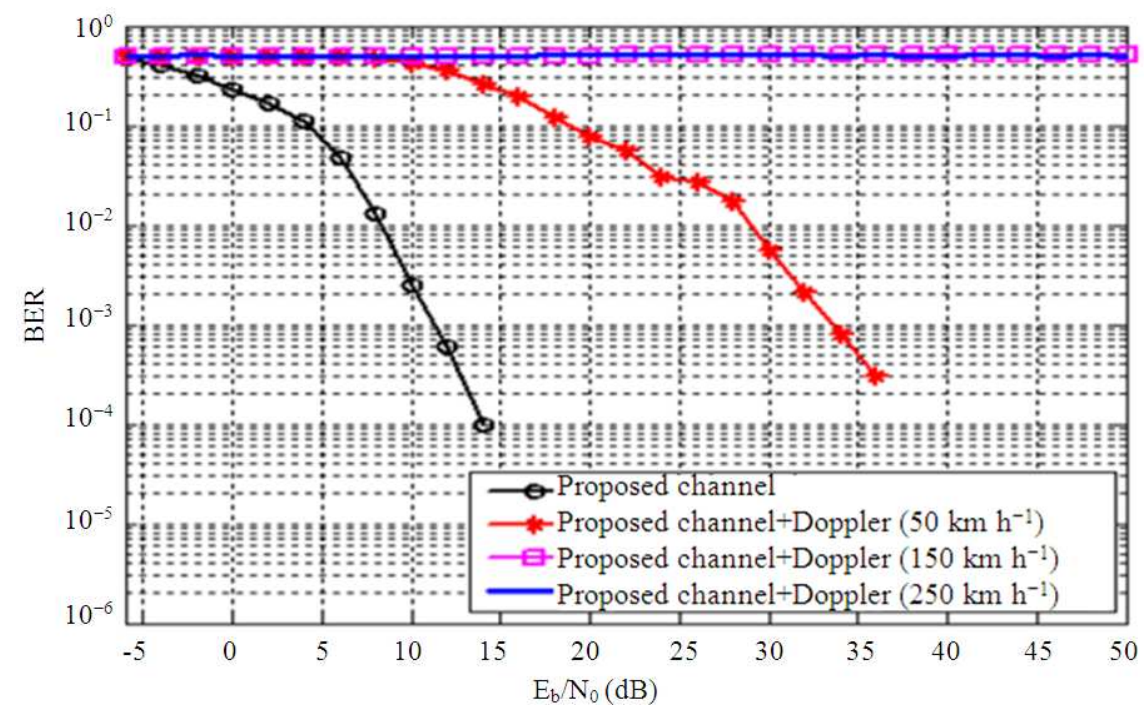

Fig. 6. BER performance of the MB-OFDM UWB system over proposed multipath channel with Doppler Shift and shadowing effect $\mathrm{X}=2$ for $200 \mathrm{Mbit} / \mathrm{s}$

\subsection{Zero-Forcing (ZF) Equalizer}

An equalizer is a compensator for Channel Distortion. For communication channels in which the channel characteristics are unknown or time-varying, optimum transmit and receive filters cannot be designed directly. For such channels, an equalizer is needed to compensate the distortion in the channel. This form of equalizer was first proposed by Robert Lucky, it applies the inverse of the channel frequency response to the received signal, to restore the signal after the channel. The coefficients are given by Equation (15):

$\mathrm{g}_{\mathrm{n}, \mathrm{i}}=\frac{1}{\mathrm{~h}_{\mathrm{n}, \mathrm{i}}}$

where, $h_{n, i}$ represents the complex response of the channel for $n$ subcarrier of $i$ symbol.

\subsection{Maximum Likelihood Estimation (ML) (Kumar et al., 2009)}

In this technique, the Carrier Frequency Offset (CFO) is first estimated statistically using a Maximum Likelihood (ML) algorithm in order to reduce ICI. Once a precise CFO estimate is obtained, it is cancelled at the receiver. This approach involves the replication of an OFDM symbol before the transmission and a comparison of the phases of each of the subcarriers between the successive symbols.
An OFDM symbol of sequence length $\mathrm{N}$ is replicated in the transmission, the receiver receives in the absence of noise, $2 \mathrm{~N}$ point, the first $\mathrm{N}$ symbols is demodulated using N-point FFT to yield the sequence $r(n)$, which is equal to Equation (16):

$r(n)=\frac{1}{N}\left[\sum_{k=-K}^{K} x_{k} H(k) e^{j 2 \pi n\left(f_{k} T+\varepsilon\right) / N}\right]$

where, $\mathrm{x}_{\mathrm{k}}$ is the complex signal modulating the $2 \mathrm{~K}+1$ subcarrier. $\mathrm{H}(\mathrm{k})$ is the channel transfer function for the kth subcarrier.

The first set of $\mathrm{N}$ symbols is demodulated using $\mathrm{N}$ point FFT we have $R_{1}(K)$ Equation (17):

$R_{1}(k)=\sum_{n-0}^{N-1} r(n) e^{-j 2 \pi f_{k} T / N}$

The second set is demodulated using another N-point FFT to yield the sequence $R_{2}(K)$ Equation (18 to 20 ):

$$
\begin{aligned}
& \mathrm{R}_{2}(\mathrm{k})=\sum_{\mathrm{n}=\mathrm{N}}^{2 \mathrm{~N}-1} \mathrm{r}(\mathrm{n}) \mathrm{e}^{-\mathrm{j} 2 \pi \mathrm{nf} \mathrm{f}_{\mathrm{k}} \mathrm{T} / \mathrm{N}} \\
& \mathrm{R}_{2}(\mathrm{k})=\sum_{\mathrm{n}=0}^{\mathrm{N}-1} \mathrm{r}(\mathrm{n}+\mathrm{N}) \mathrm{e}^{-\mathrm{j} 2 \pi \mathrm{nn} \mathrm{f}_{\mathrm{k}} \mathrm{T} / \mathrm{N}} \\
& \mathrm{R}_{2}(\mathrm{k})=\mathrm{R}_{1}(\mathrm{k}) \mathrm{e}^{\mathrm{j} 2 \pi \varepsilon}
\end{aligned}
$$


The frequency offset is the phase difference between the sequences $\mathrm{R}_{1}(\mathrm{k})$ and $\mathrm{R}_{2}(\mathrm{k})$. Adding the White Gaussian Noise we get Equation (21 and 22):

$$
\begin{aligned}
& \mathrm{Y}_{1}(\mathrm{k})=\mathrm{R}_{1}(\mathrm{k})+\mathrm{W}_{1}(\mathrm{k}) \\
& \mathrm{Y}_{2}(\mathrm{k})=\mathrm{R}_{1}(\mathrm{k}) \mathrm{e}^{\mathrm{j} 2 \pi \varepsilon}+\mathrm{W}_{2}(\mathrm{k})
\end{aligned}
$$

The CFO can be estimated using ML algorithm and is equal to Equation (23):

$$
\hat{\varepsilon}=\left(\frac{1}{2 \pi}\right) \tan ^{-1}\left\{\frac{\sum_{\mathrm{k}=-\mathrm{K}}^{\mathrm{K}} \operatorname{Im} \mathrm{ag}\left[\mathrm{Y}_{2}(\mathrm{k}) \mathrm{Y}_{1}^{*}(\mathrm{k})\right]}{\sum_{\mathrm{k}=-\mathrm{K}}^{\mathrm{K}} \operatorname{Re} \mathrm{al}\left[\mathrm{Y}_{2}(\mathrm{k}) \mathrm{Y}_{1}^{*}(\mathrm{k})\right]}\right\}
$$

Once the frequency offset is known, the ICI distortion in the data symbols is reduced by multiplying the received symbols with a complex conjugate of the frequency shift and applying the FFT.

\subsection{Extended Kalman Filter (Kumar et al., 2009)}

The Kalman Filter was originally designed for solving linear systems in white Gaussian noise, for the nonlinear systems, there is a method called Extended Kalman Filter (EKF), based on regular Kalman Filter, the idea is to linearize the system and then use the same method of Kalman Filter (Kumar et al., 2009). As the ML estimation, we have two stages in the EKF to mitigate the ICI effect: the offset estimation and the offset correction.

The observation equation is the Equation (13):

$$
y(n)=z(n) e^{j 2 \pi \varepsilon \frac{n}{N}}+w(n)
$$

To estimate the using an EKF in each OFDM frame, the first order state equation is built as Equation (24):

$$
\hat{\varepsilon}(n)=\hat{\varepsilon}(n-1)
$$

Assume $\mathrm{N}_{\mathrm{p}}$ preambles preceding the data symbols in each frame are used as training sequence; the algorithm for computation of frequency offset for $\mathrm{N}_{\mathrm{p}}$ iterations is (Kumar et al., 2009):

- Initialize estimate $\widehat{\varepsilon}(0)$ and corresponding state error $\mathrm{P}(0)$

- Compute the $\mathrm{H}(\mathrm{n})$, derivate of $\mathrm{y}(\mathrm{n})$

- Compute the time average Kalman gain k(n) using the error variance $\mathrm{P}(\mathrm{n}-1), \mathrm{H}(\mathrm{n})$ and the variance $\sigma^{2}$ of the AWGN w(n)
- Compute the estimate using $\mathrm{y}(\mathrm{n}), \mathrm{z}(\mathrm{n}), \mathrm{k}(\mathrm{n})$ and $\widehat{\varepsilon}(\mathrm{n}-1)$

- Compute the state error $\mathrm{P}(\mathrm{n})$ with Kalman gain $\mathrm{k}(\mathrm{n})$, $\mathrm{H}(\mathrm{n})$ and the previous error $\mathrm{P}(\mathrm{n}-1)$

- If $\mathrm{n}$ is less than $\mathrm{N}_{\mathrm{p}}$, increment by 1 and go to step 2, otherwise stop

The ICI distortion in the data symbols $x(n)$ can then be cancelled by multiplying the received data symbols $y(n)$ by complex conjugate of the estimated frequency offset and applying FFT.

\section{RESULTS AND DISCUSSION}

The performance of the proposed system (Fig. 2) for $200 \mathrm{Mbit} / \mathrm{s}$ is evaluated. Figure 7 shows the evaluation of $B E R$ versus $E_{b} / N_{0}$ over Proposed multipath channel for two paths and in the presence of shadowing effect and Doppler shift for different value of speed $\mathrm{V}=[50,150,250] \mathrm{Km} \tilde{\mathrm{h}} 1$ with and without Zero Forcing equalizer $(\mathrm{ZF})$. The $\mathrm{E}_{\mathrm{b}} / \mathrm{N}_{0}$ is varying between -6 and $50 \mathrm{~dB}$.

It is observed in the Fig. 7 that for lower value of relative speeds $\left(50,150 \mathrm{~km} \mathrm{~h}^{-1}\right)$, Zero Forcing (ZF) equalizer gives good results in terms of BER and reduces Doppler Effect. However, for higher value of speed (250 $\mathrm{km} \mathrm{h}^{-1}$ ), the ZF equalizer does not offer much improvement in performance, it is not sufficient.

The goal in this article is to find a compact model to estimate and reduce the effect of ICI in the MB-OFDM UWB system and make a compromise between the simplicity of implementation and good performance. The simulation result of $\mathrm{ZF}$ equalizer shows that the performance is improved only under the low mobile environments and it doesn't completely cancel the ICI effect, therefore two ICI reduction algorithms are explored for mitigation of the ICI namely, the Maximum Likelihood (ML) estimation and the Extended Kalman Filter (EKF). These ICI cancellation methods are used with $\mathrm{ZF}$ equalizer to increase the performance of the MB-OFDM UWB system (Fig. 8).

Figure 9-11 show the simulation results of BER performance versus $E_{b} / N_{0}$ for the two ICI reduction algorithms (ML and EKF) over the proposed multipath channel with the shadowing $X=2$ and Doppler shift for different values of speed $\mathrm{V}=[50$, $150,250] \mathrm{Km} \mathrm{h}^{-1}$, compared to the simulation results over the proposed multipath channel in the presence of Doppler shift for the $\mathrm{ZF}$ equalizer. The $\mathrm{E}_{\mathrm{b}} / \mathrm{N}_{0}$ is varying between -6 and $50 \mathrm{~dB}$. 


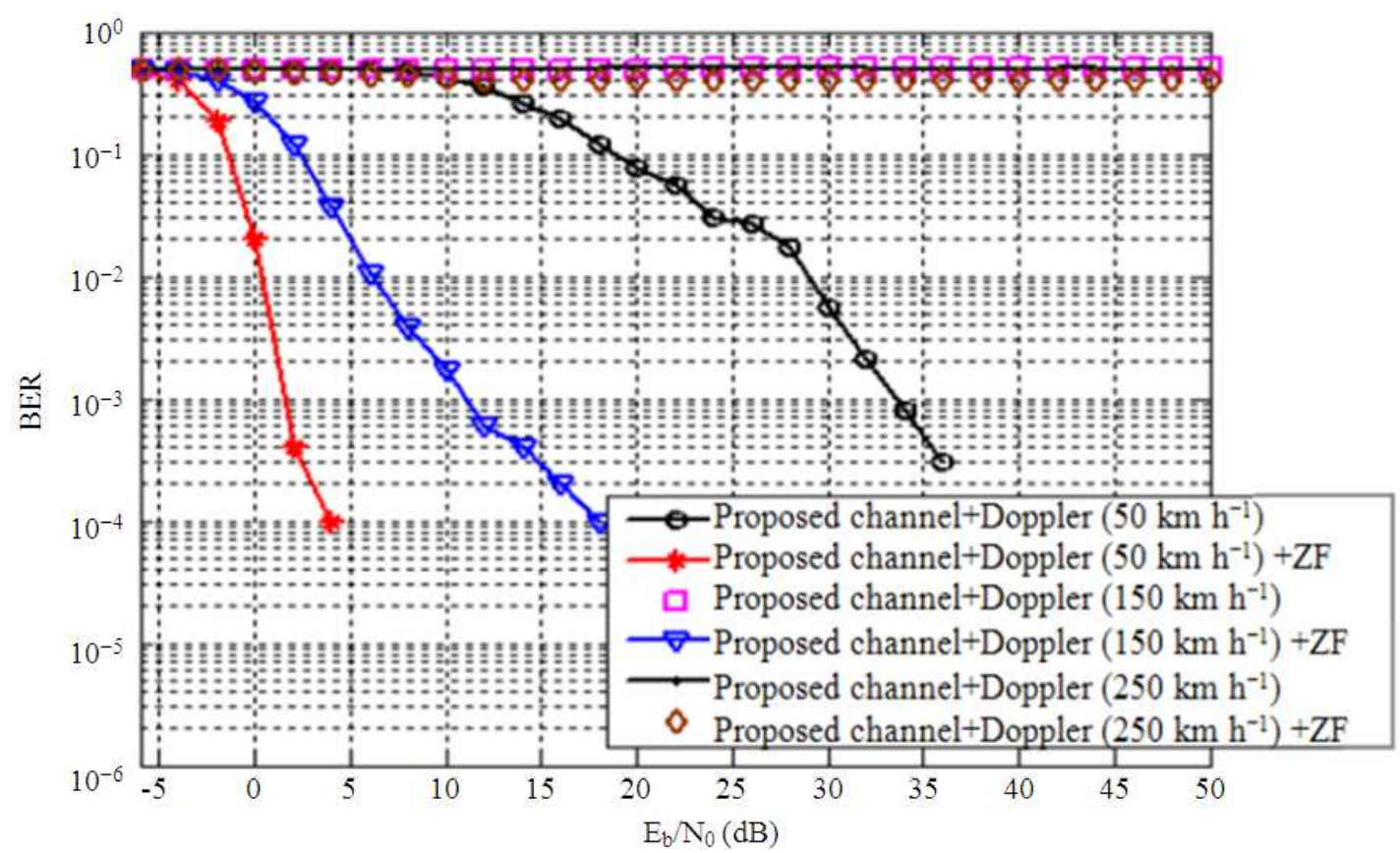

Fig. 7. BER performance for the MB-OFDM UWB system over proposed multipath channel with and without ZF equalizer for three different speeds for $200 \mathrm{Mbit} / \mathrm{s}$

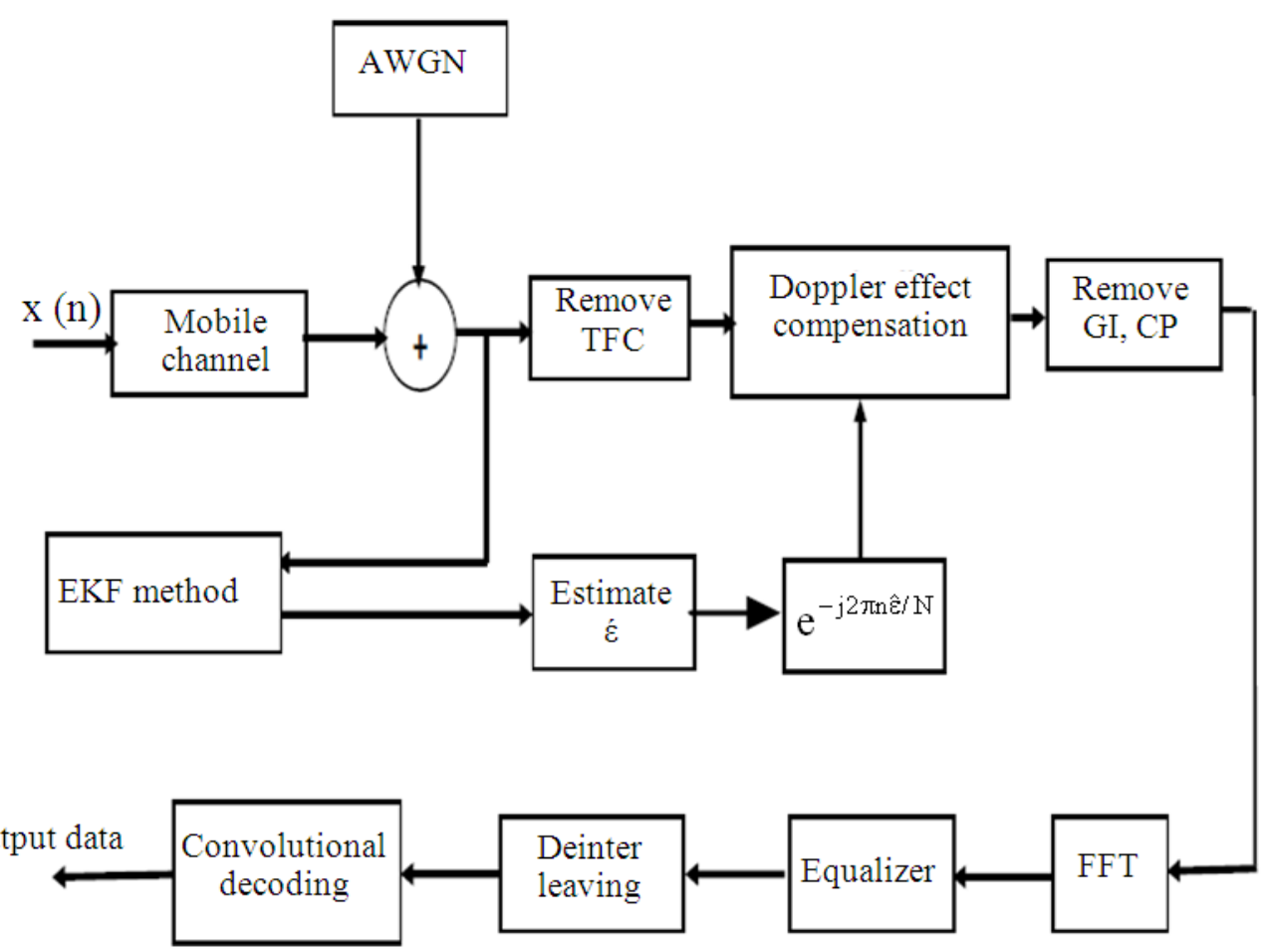

Fig. 8. Receiver block diagram for the MB-OFDM UWB system with ICI reduction methods and channel equalization 


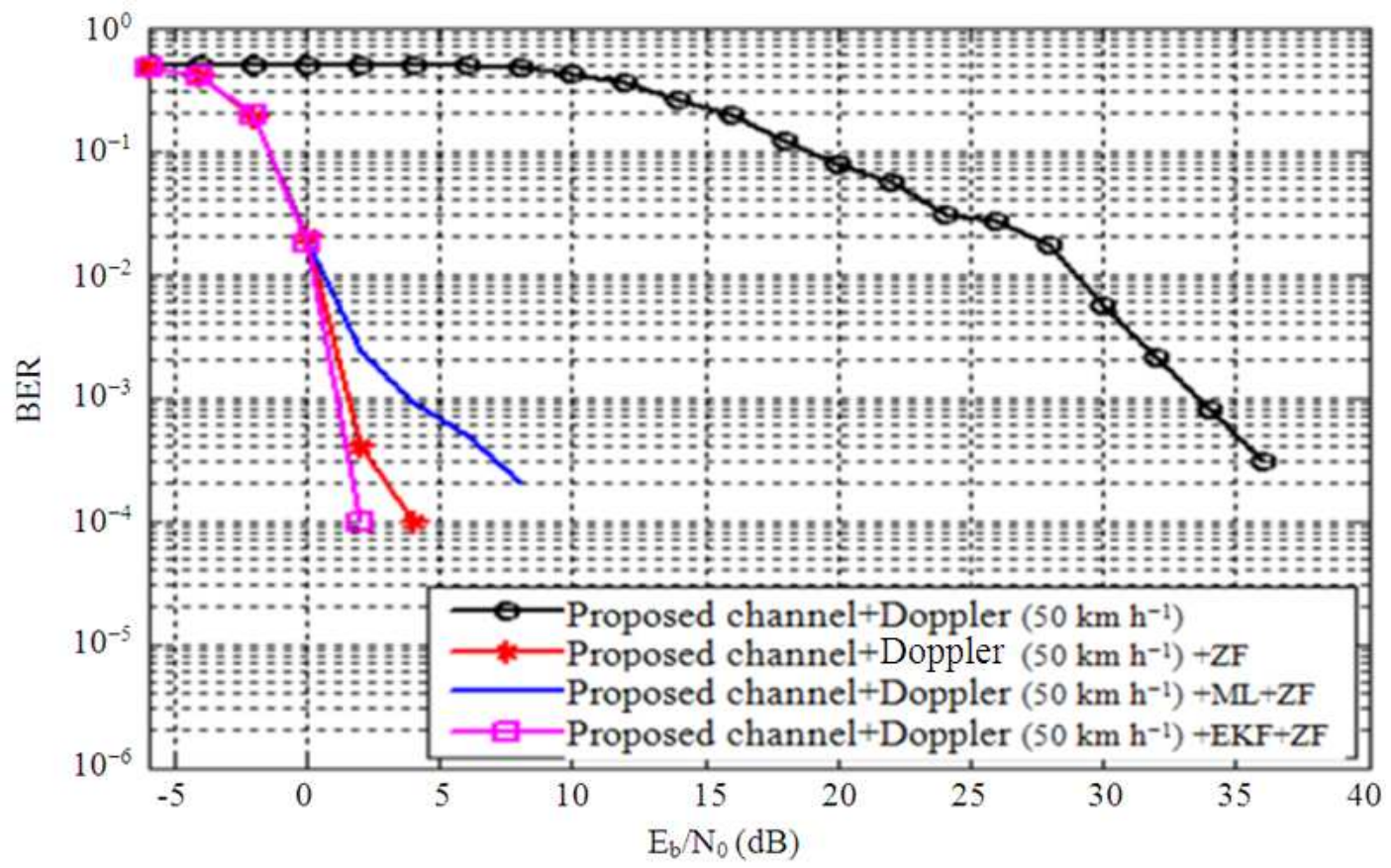

Fig. 9. Performance comparison of various ICI reduction algorithms for the MB-OFDM UWB system over Proposed Multipath Channel for $\mathrm{V}=50 \mathrm{Km} \mathrm{h}^{-1}$

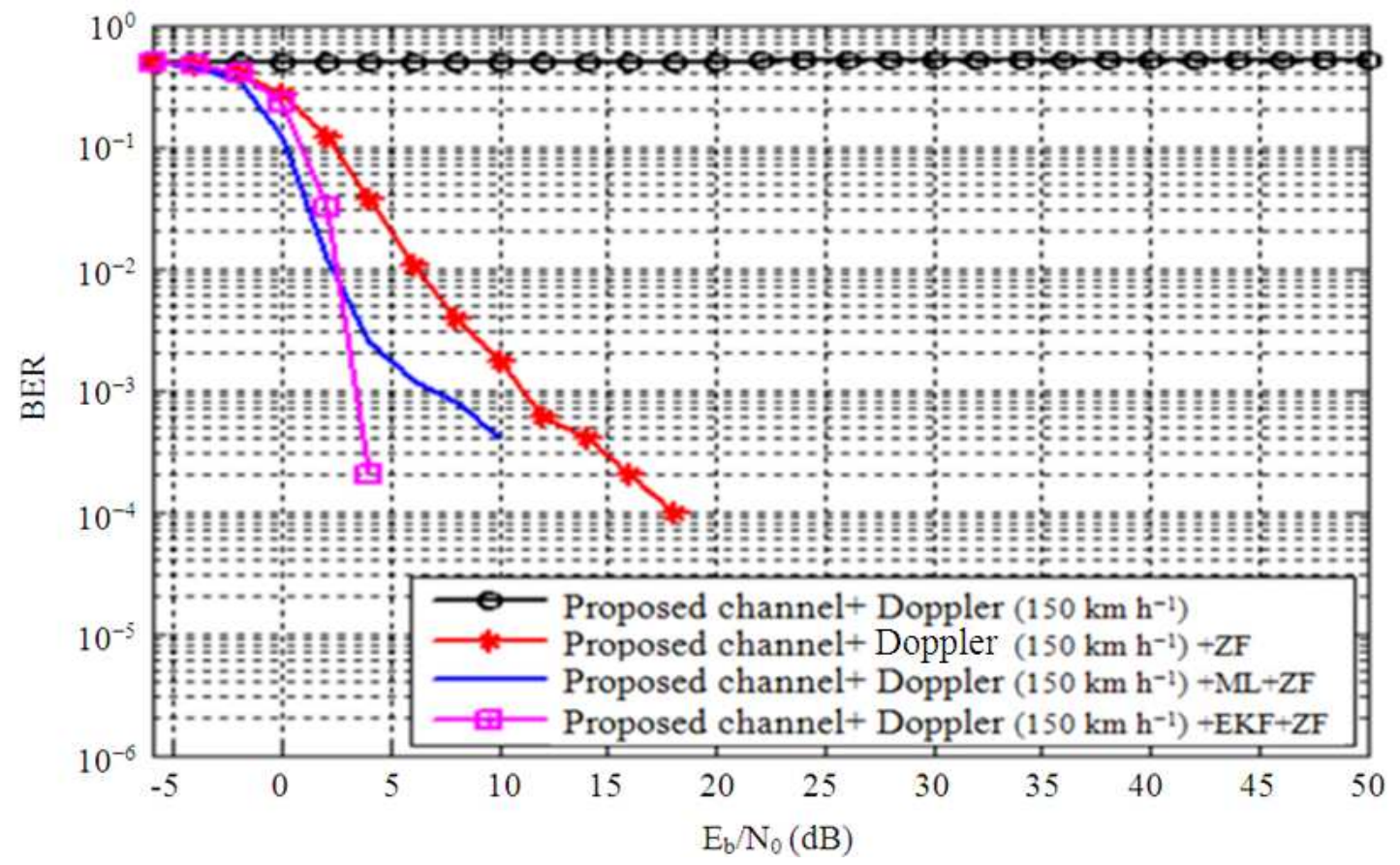

Fig. 10. Performance comparison of various ICI reduction algorithms for the MB-OFDM UWB system over Proposed Multipath Channel for $\mathrm{V}=150 \mathrm{Km} \mathrm{h}^{-1}$ 


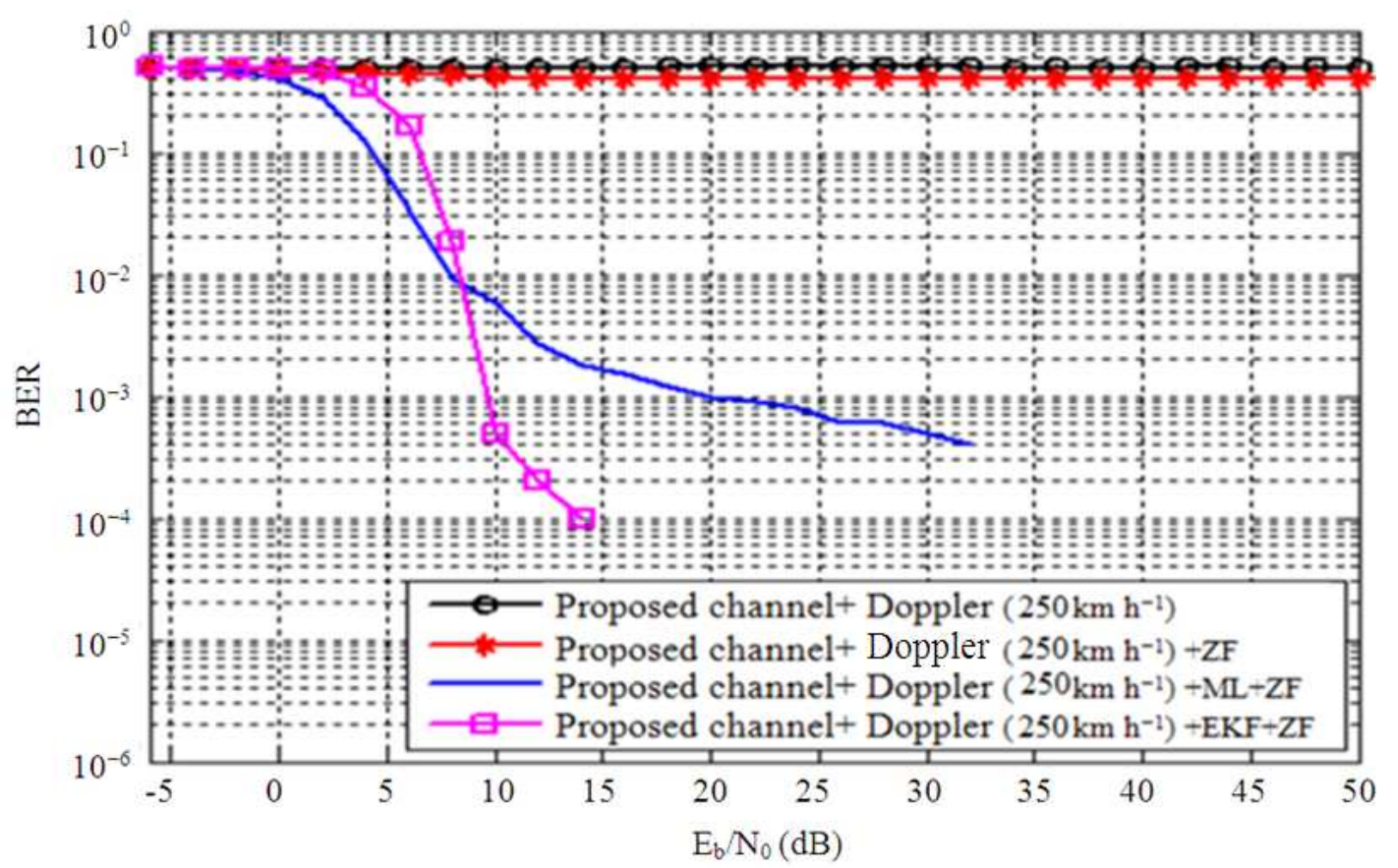

Fig. 11. Performance comparison of various ICI reduction algorithms for the MB-OFDM UWB system over Proposed Multipath Channel for $\mathrm{V}=250 \mathrm{Km} \mathrm{h}^{-1}$

According to the figures, it is observed that for speeds $\left(50 \mathrm{Km} \mathrm{h}^{-1}, 150 \mathrm{Km} \mathrm{h}^{-1}\right)$, the ML and EKF methods give good performance in term of BER as ZF equalizer. For V $=250 \mathrm{~km} \mathrm{~h} 1$, the EKF method has better BER than ML estimation better than $\mathrm{ZF}$ equalizer. For a particular case at $\mathrm{BER}=10^{-3}$, the EKF method gives best results, we have $\mathrm{E}_{\mathrm{b}} / \mathrm{N}_{0}=9.617 \mathrm{~dB}$, compared to ML estimation, we need $\mathrm{E}_{\mathrm{b}} / \mathrm{N}_{0}=20 \mathrm{~dB}$. The EKF does perform extremely well and gives a significant boost to performance and it is more efficient than ML estimation.

\section{CONCLUSION}

In this study, we have proposed the MB-OFDM UWB system as a good candidate technology to ensure V2I communication; indeed, the proposed system offers a high data rate (over $100 \mathrm{Mbit} / \mathrm{s}$ ). The theoretical formulas BER over AWGN and Rayleigh channels are compared to the simulation results for the MB-OFDM UWB system and it improves that the simulation part is consistent with the theoretical formulas.

The performance of the proposed system in the presence of Doppler shift between the transmitter and the receiver has been studied in term of BER. Inter-
Carrier Interference (ICI) which results from the frequency offset degrades the performance of the MBOFDM UWB system. Three methods were explored in this article for mitigation of the ICI, the ZF equalizer, the EKF and ML estimation. The EKF is proposed as the good ICI reduction method, significant gains in performance can be achieved using the EKF method for large and low speeds and therefore frequency offset values.

\section{REFERENCES}

Albarazi, K., U. Mohammad and N. Al-holou, 2011. Doppler shift impact on vehicular ad-hoc networks. Canadian J. Multimedia Wireless Networks, 2: 4656.

Alvin, S., 2011. Efficient safety message dissemination for cooperative collision warning via context modelling. PhD. Thesis, Queensland University of Technology, Australia.

Bahai, A.R.S., B.R. Saltzberg and M. Ergen, 2004. Multi-Carrier Digital Communications: Theory and Applications of OFDM. 1st Edn., Springer, New York, ISBN-10: 0387225757, pp: 411. 
Bai, F., H. Krishnan, T. Elbatt and G. Holland, 2012. Towards characterising and classifying communication-based automotive applications from a wireless networking perspective. Int. J. Vehicle Autonomous Syst., 10: 165-197. DOI: 10.1504/IJVAS.2012.051267

Chantaksinopas, I., W. Lee, A. Prayote and P. Oothongsap, 2012. Delay-sensitive applications in vanet and seamless connectivity: The limitation of UMTS network. Int. J. Comput. Sci. Netw. Sec., 12: 54-61.

Fawaz, K., A. Ghandour, M. Olleik and H. Artail, 2010. Improving reliability of safety applications in vehicle ad hoc networks through the implementation of a cognitive network. Proceedings of the 17th International Conference on Telecommunications, Apr. 4-7, IEEE Xplore Press, pp: 798-805. DOI: 10.1109/ICTEL.2010.5478817.

Fernandes, J.R. and D. Wentzloff, 2010. Recent advances in IR-UWB transceivers: An overview. Proceedings of the IEEE International Symposium on Circuits and Systems, May 30-Jun. 2, IEEE Xplore Press, Paris, pp: 3284-3287. DOI: 10.1109/ISCAS.2010.5537916

Kumar, A. and R. Pandey, 2013. A spectrally efficient intercarrier interference reduction scheme for orthogonal frequency division multiplexing systems in low signal-to-noise ratio environment. IETE J. Res., 59: 74-82. DOI: 10.4103/0377-2063.110634

Kumar, B.S., K.R. S. Kumar and R. Radhakrishnan, 2009. An efficient inter carrier interference cancellation schemes for OFDM systems. Int. J. Comput. Sci. Inform. Sec., 6: 141-148.

Mohapatra, S. and S. Das, 2009. Performance enhancement of OFDM system with ICI reduction. Proceedings of the World Congress on Engineering Technique, Jul. 1-3, London, U.K., pp: 459-462.

Molisch, A.F., K. Balakrishnan, C.C. Chong, S. Emami and A. Fort et al., 2004. IEEE 802.15.4a channel model-final report. Working Group for Wireless Personal Area Networks.
Petkovic, D.P. and M. Bjelica, 2007. Mobile broadcasting technologies: A comparative analysis. Proceedings of the 15th Telecommunications Forum Serbia, Nov. 20-22, Belgrade, pp: 733-736.

Platzner, M., J. Teich and N. When, 2010. Dynamically reconfigurable systems: architectures, Design Methods and Applications. 1st Edn., Springer, New York, ISBN-13: 978-90-481-3484-7, pp: 295.

Rahman, M., P.K. Dey and M.F.R. Ur Rashid, 2012. Improved ICI self cancellation scheme for phase rotation error reduction in OFDM system. Int. J. Inform. Electron. Eng., 2: 509-511. DOI: 10.7763/IJIEE.2012.V2.149

Sharma, L.K., H. Saini, T.C. Panda and H.N. Pratihari, 2010. Taxonomy of cell planning. Int. J. Rev. Comput., 3: 66-74.

Sreekanth, N. and M.N. GiriPrasad, 2012. BER analysis of mitigation of ICI through ICI self cancellation scheme in OFDM systems. Int. J. Comput. Netw. Wireless Commun., 2: 298-304.

Wang, Q., J. Hu and J. Zhang, 2012. Performance evaluation of information propagation in vehicular ad hoc network. Intell. Transport Syst., 6: 187-196. DOI: 10.1049/iet-its.2010.0131

Win, M.Z., D. Dardari, A.F. Molisch, W. Wiesbeck and J. Zhang, 2009. History and applications of UWB. Proc. IEEE, 97: 198-204. DOI: 10.1109/JPROC.2008.2008762

Zhao, Y. and S.G. Haggman, 1996. Sensitivity to Doppler shift and carrier frequency errors in OFDM systems-the consequences and solutions. Proceedings of the IEEE 46th Vehicular Technology Conference, Mobile Technology for the Human Race, Apr. 28-May 1, IEEE Xplore Press, Atlanta, GA., $\quad$ pp: 1564-1568. DOI: 10.1109/VETEC.1996.504021 